PANCREAS

\title{
Dynamic imaging of pancreatic diseases by contrast enhanced coded phase inversion harmonic ultrasonography
}

\author{
M Kitano, M Kudo, K Maekawa, Y Suetomi, H Sakamoto, N Fukuta, R Nakaoka, T Kawasaki
}

See end of article for authors' affiliations ....................

Correspondence to: Dr M Kitano, Division of Gastroenterology and Hepatology, Department of Internal Medicine, Kinki University School of Medicine, 377-2, Ohno-Higashi, Osaka-Sayama, 589-8511, Japan; m-kitano@ med.kindai.ac.jp

Accepted for publication 10 December 2003
Background: Coded phase inversion harmonic ultrasonography, a newly available sonographic technique, enables visualisation of slow flow in minute vessels in a real time fashion with the use of a sonographic contrast agent containing monosaccharide. Our purpose was to employ this novel technique to observe microvessels in pancreatic tumours.

Subjects and methods: Sixty five patients with suspicious pancreatic tumours received contrast enhanced coded phase inversion harmonic ultrasonography, contrast enhanced computed tomography, and endosonography. Final diagnoses based on histological findings were pancreatic ductal carcinomas in 49 patients, inflammatory pseudotumours with chronic pancreatitis in seven, and endocrine tumours in nine. For contrast enhanced coded harmonic ultrasonography, Levovist, a contrast agent, was injected intravenously as a bolus. When the first microbubble signal appeared in the pancreas, images of the ideal scanning plane were displayed in a real time continuous fashion (vessel images). Subsequently, interval delay scanning (perfusion images) was taken to demonstrate parenchymal flow. Tumour vascularity was evaluated by using the two types of imaging. Sensitivities for depicting pancreatic tumours were compared between three examinations.

Results: Contrast enhanced ultrasonography demonstrated tumour vessels in $67 \%$ of pancreatic ductal carcinomas, although most were relatively hypovascular compared with the surrounding pancreatic tissue. The vascular patterns of tumours obtained by contrast enhanced ultrasonography were closely correlated with those obtained by contrast enhanced computed tomography. Values for sensitivity in depicting pancreatic tumours of $2 \mathrm{~cm}$ or less in size were $68 \%$ for contrast enhanced computed tomography, $95 \%$ for endosonography, and $95 \%$ for contrast enhanced ultrasonography.

Conclusion: Contrast enhanced coded phase inversion harmonic ultrasonography successfully visualised fine vessels in pancreatic tumours and may play a pivotal role in the depiction and differential diagnosis of pancreatic tumours.
$\mathrm{T}$ he diagnosis of pancreatic cancers, particularly their differential diagnosis from chronic pancreatitis, has often been difficult, even when a combination of various imaging modalities such as ultrasonography (US), magnetic resonance cholangiopancreatography (MRCP), endoscopic retrograde cholangiopancreatography (ERCP), endosonography (EUS), contrast enhanced computed tomography (CT), and catheterised angiography are employed. Contrast enhanced Doppler sonography has been proposed as a valuable technique for the diagnosis of hepatic and pancreatic tumours. ${ }^{1-9}$ However, there are still inherent limitations for observation of real time vessel image because of artefacts such as blooming or overpainting. Coded phase inversion harmonic imaging is a newly available sonographic technique which is based on a combination of phase inversion harmonics and coded technology. ${ }^{10-15}$ With the use of a microbubble contrast agent (a suspension of monosaccharide microparticles in sterile water), it depicts signals from microbubbles in very slow flow without Doppler related artefacts, and enables visualisation of slow flow in microscopic vessels.

In this study, we assess the usefulness of coded phase inversion imaging for depiction and differential diagnosis of pancreatic tumours compared with contrast enhanced CT and EUS.

\section{SUBJECTS AND METHODS}

Subjects

The study was performed with the approval of our institutional review board. Between March 2001 and August 2003,
65 consecutive patients suspected of having a pancreatic mass underwent contrast enhanced coded harmonic US, contrast enhanced two phase CT, and EUS at our institution. All patients underwent the three examinations when a pancreatic mass was suspected (or detected) with one of the following: plain or contrast enhanced one phase CT, fundamental B-mode US, MRCP, or ERCP. We did not conduct contrast enhanced harmonic US as a screening test in patients who were never suspected of having a pancreatic tumour by any examination. Pancreatic masses in 39 patients were suspected from conventional US or CT at other clinics. The other 26 patients suspected of having a pancreatic mass had undergone B-mode US, CT, MRCP, or ERCP as a screening device at our institution or had symptoms. The gold standard in the present study was histological findings obtained by EUS guided fine needle aspiration biopsy (EUSFNA) $(\mathrm{n}=34)$, surgery $(\mathrm{n}=20)$, biopsy of liver metastases $(n=5)$, and autopsy $(n=6)$. The diagnoses of the seven tumours were also based on a clinical course for more than 12 months, after excluding malignancy by EUS-FNA. As they did not change in size throughout the observation period, they were diagnosed as inflammatory pseudotumours with chronic pancreatitis. Thus the final diagnoses of pancreatic

\footnotetext{
Abbreviations: US, ultrasonography; MRCP, magnetic resonance cholangiopancreatography; ERCP, endoscopic retrograde cholangiopancreatography; CT, computed tomography; EUS, endosonography; FNA, fine needle aspiration
} 
tumours included 49 patients with pancreatic ductal carcinomas, seven patients with inflammatory pseudotumours related to chronic pancreatitis, and nine patients with endocrine tumours.

\section{Contrast enhanced US examination}

To minimise the procedural variations, contrast enhanced US was performed by the same sonographer (KM) using the same examination protocol. The technique had been established by the sonographer (KM) who had conducted contrast enhanced US in more than 1000 patients before beginning the present study. The sonographer (KM) was informed that a pancreatic mass had been suspected by other imaging modalities but was blinded to the location and findings of the tumour. Ultrasonographic equipment with a 2-4 MHz curved array wide band transducer, GE LOGIQ 9, and 700 EXPERT Series units (General Electric Medical Systems, Milwaukee, Wisconsin, USA) was used for coded phase inversion imaging. ${ }^{10-15}$ The acoustic power was set at the default setting with a mechanical index of 0.6-0.8. After detecting an abnormality such as a nodule, swelling, or stenosis of the main duct in the pancreas on fundamental B-mode US, the sonographer conducted contrast enhanced US, displaying the ideal scanning plane of the lesion. When the first microbubble signal appeared in the tumour after bolus injection of $2.5 \mathrm{~g}$ of Levovist (a suspension of monosaccharide microparticles, $6 \mathrm{ml}$ of a $400 \mathrm{mg} / \mathrm{ml}$ concentration; Schering AG, Berlin, Germany), the patient was instructed to hold his/her breath. Images of the ideal scanning plane were displayed in real time by slightly changing the scanning plane to portray the whole area of the tumour (vessel image). In addition to real time continuous imaging of the tumour vessels, interval delay scanning was performed to demonstrate tumour parenchymal flow in the blood pool phase (perfusion image, five seconds of interval time, less than 90 seconds after injection of Levovist). The entire examination was stored in a recording system and reviewed by two readers who were absent during the US examination and completely blinded to the results of the previous investigations, including fundamental B-mode US, CT, MR, and ERCP. The reviewers assessed the vascular patterns of the tumours.

\begin{tabular}{lll} 
Type & $\begin{array}{c}\text { Vessel } \\
\text { image }\end{array}$ & $\begin{array}{c}\text { Perfusion } \\
\text { image }\end{array}$ \\
\hline$I(n=15)$ &
\end{tabular}

Figure 1 Classification of vascular patterns of pancreatic tumours. Type I: no vessels on the vessel image and no enhancement on the perfusion image. Type II: few vessels on the vessel image and heterogeneous enhancement in the hypovascular area on the perfusion image. Vascularity is less than in the surrounding pancreatic tissue. Type III: similar vascularity to the surrounding pancreatic tissue on the vessel image and homogenous isovascular enhancement on the perfusion image. Type IV: abundant vessels on the vessel image and hypervascular enhancement on the perfusion image.

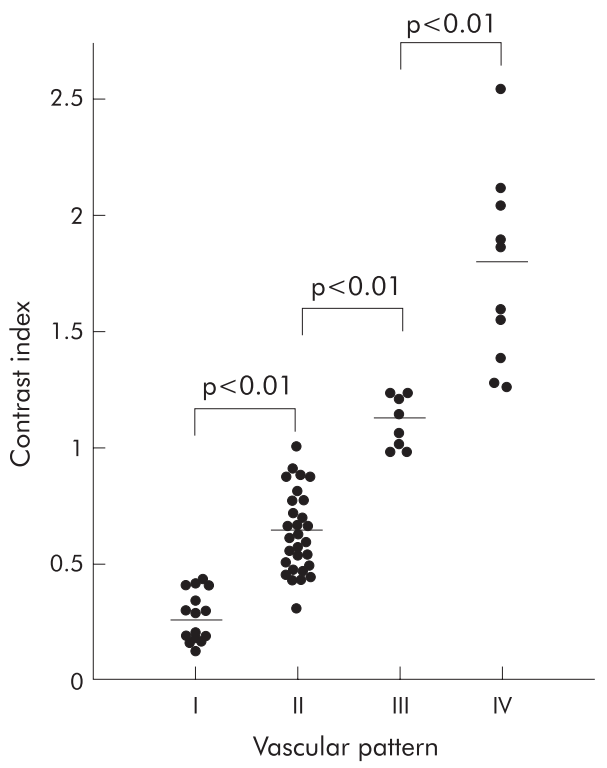

Figure 2 Contrast indices of the four types of contrast enhanced ultrasonography classified tumours. $\mathrm{p}<0.05$ was considered significant.

They had experience of reading images of contrast enhanced

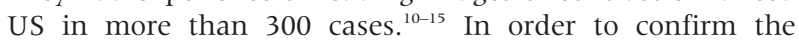
accuracy of the reviewers' evaluations, the contrast index was calculated on the perfusion images of the coded phase inversion harmonic US (contrast index = mean echo intensity in the tumour/mean echo intensity in the adjacent pancreatic tissue).

\section{Contrast enhanced CT}

Contrast enhanced two phase CT (Toshiba X-vigor, Toshiba Medical System, Japan) was imaged 30 and 180 seconds after the beginning of injection of $100 \mathrm{ml}$ of contrast media (Optiray 320; Yamanouchi Pharmaceutical, Tokyo, Japan) into the antecubital vein with a $5.0 \mathrm{~mm}$ slice thickness. Images were reviewed by two readers who were blinded to the US and pathological findings.

\section{Endosonography}

EUS was performed by two qualified (from the Japanese Gastroenterological Endoscopic Society) endoscopists (MK, RN), using an Olympus GF-UC240P-AL5 for endoscopy and an Aloka ProSound-5500 for image analysis. EUS-FNA was performed in 34 patients. The needle for the aspiration biopsy was an Olympus NA-10J-1 or an Olympus NA-11J-KB.

\section{Statistical analysis}

Sensitivities for depicting pancreatic tumours of $2 \mathrm{~cm}$ or less and those of more than $2 \mathrm{~cm}$ in size were calculated from contrast enhanced US, contrast enhanced CT, and EUS. The $\chi^{2}$ test for non-parametric data was performed to compare the sensitivities for depiction of tumours between the three examinations. Scheffe's multiple comparison test was used to compare contrast indices between the four types of contrast enhanced US classified tumours. A p value of $<0.05$ was considered significant.

\section{RESULTS}

Normal pancreatic tissue showed several vessels distributed homogeneously on the vessel image and a homogeneous stain on the perfusion image. On the basis of the density of vessels on the vessel image and enhancement on the perfusion image relative to the surrounding pancreatic tissue, 

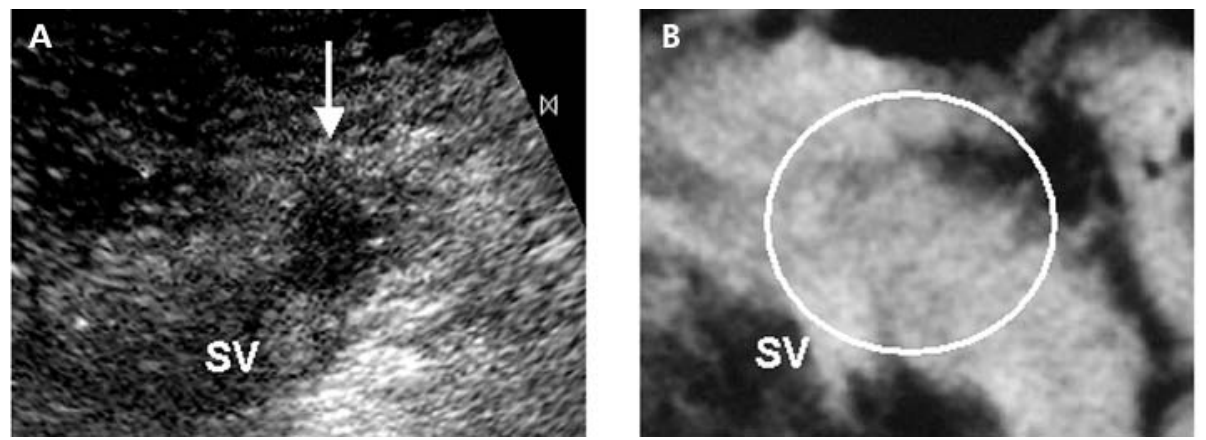

Figure 3 Typical case of a type I tumour. A 43 year old man with a pancreatic ductal cancer found by contrast enhanced ultrasonography (US). (A) Perfusion image of contrast enhanced US shows a hypovascular tumour of $1.3 \mathrm{~cm}$ in diameter adjacent to the splenic vein (SV). (B) Contrast enhanced computed tomography failed to depict the tumour.

the image patterns of the pancreatic tumours were classified into the following four types (fig 1): type I, no vessels on the vessel image and hypovascular enhancement on the perfusion image; type II, low density of vessels on the vessel image and slight heterogeneous enhancement in the hypovascular area on the perfusion image; type III, similar density of vessels to the surrounding tissue on the vessel image and homogenous isovascular enhancement on the perfusion image; and type IV, high density of vessels on the vessel image and hypervascular enhancement on the perfusion image. The contrast indices of types I, II, III, and IV were mean 0.28 (SD 0.10), $0.64(0.17), 1.11(0.11)$, and 1.76 $(0.42)$, respectively (fig 2 ). The contrast indices were significantly different among the four types. A close correlation was demonstrated between the contrast index and the image pattern (fig 2).

Fourteen of 15 tumours classified as type I were ductal carcinomas (fig 3). Only one of 15 tumours was an endocrine tumour. All 29 tumours classified as type II were ductal carcinomas. On the vessel images of type II tumours, several vessels surrounding the tumour and flowing from the peripheral part to the centre of the tumour were depicted. The perfusion images of type II tumours showed a heterogeneous network-like stain of the tumour although it appeared as a hypovascular tumour relative to the surrounding tissue (fig 4). Seven inflammatory pseudotumours caused by chronic pancreatitis showed the type III pattern in which there were no differences in the images (vessel and perfusion) between the inside and outside of the tumours (fig 5). Only one of the 49 ductal carcinomas showed the type III pattern. Seven of the 10 tumours classified as type IV were endocrine tumours (fig 6). There were rich vessels on the vessel image and remarkable enhancement on the perfusion image of the endocrine tumours (fig 6). The other three tumours classified as type IV were pancreatic carcinomas. On contrast enhanced US, tumour vessels were visualised in 33
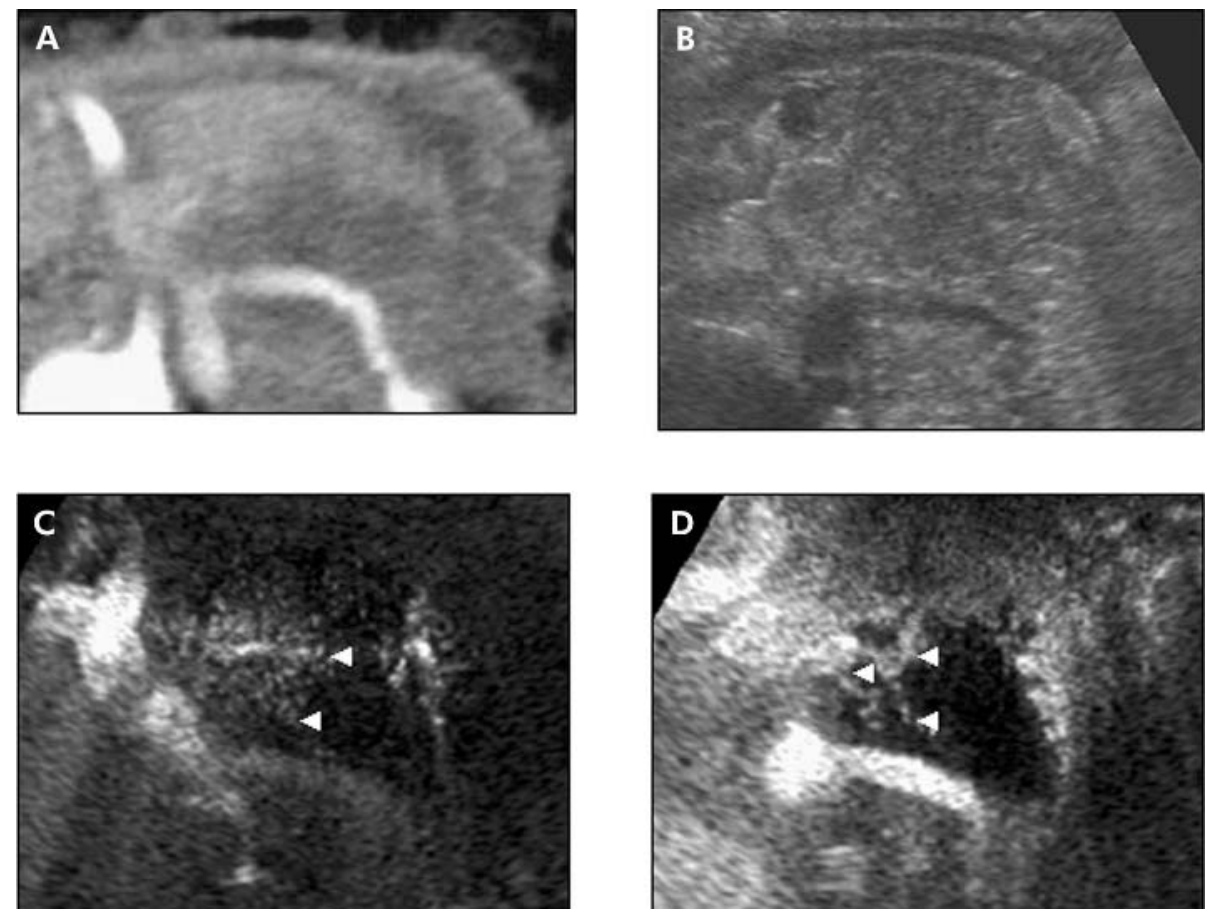

Figure 4 Typical case of a type II tumour. A 71 year old man with a pancreatic ductal cancer. (A) Contrast enhanced computed tomography shows a low dense mass in the pancreatic body to the tail. (B) Fundamental B-mode ultrasonography (US) shows the swollen pancreas. However, the margin of the tumour is unclear. (C) Vessel image of contrast enhanced US. The architecture of the vessels (arrowheads) in the tumour was clearly observed. (D) Perfusion image of contrast enhanced US. A hypovascular tumour with heterogeneous network-like enhancement (arrowheads) was clearly depicted. 

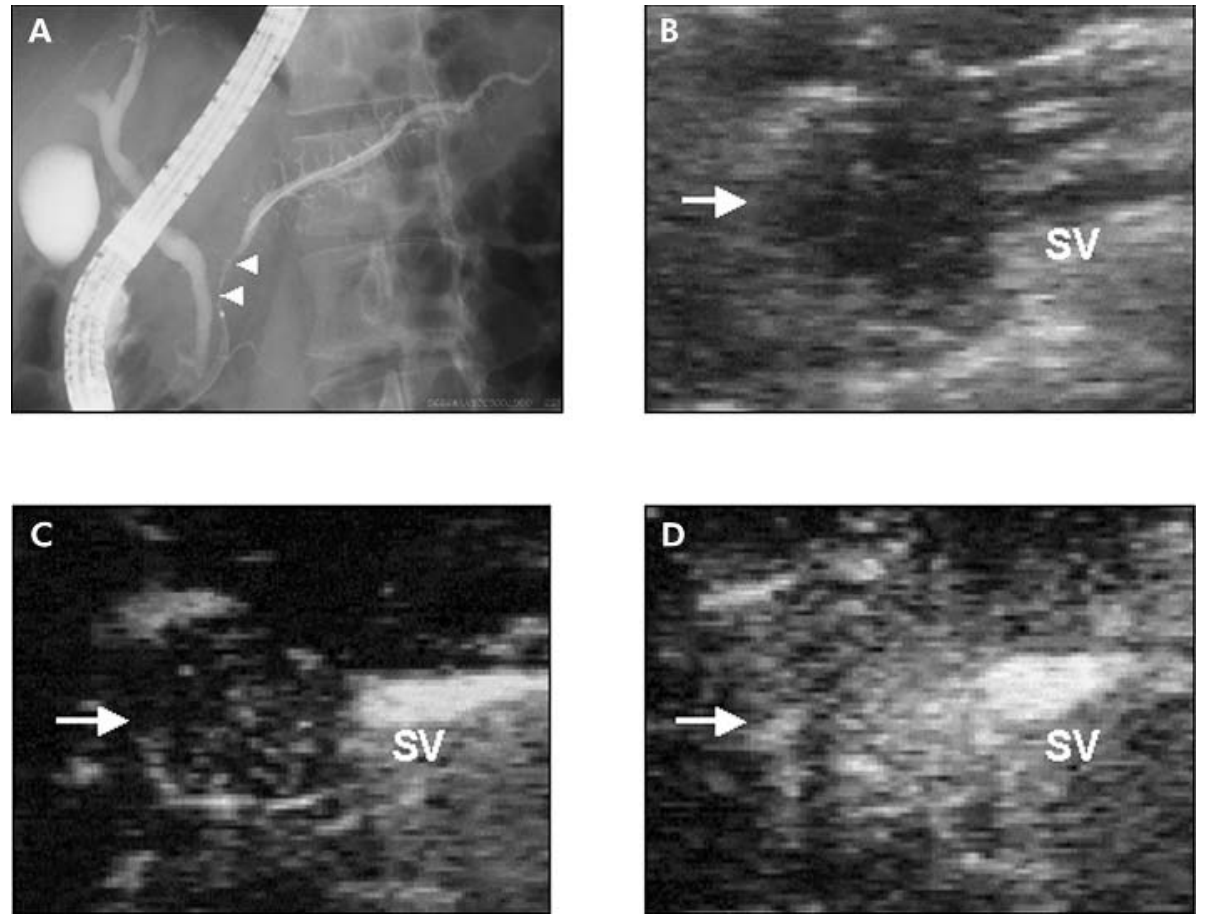

Figure 5 Typical case of a type III tumour. A 59 year- old man with an inflammatory pseudotumour with chronic pancreatitis. (A) Endoscopic retrograde cholangiopancreatography shows stenosis of the main duct of the pancreatic head (arrowheads). (B) Fundamental B-mode ultrasonography (US) shows a low echoic mass (arrow) in the pancreatic head. (C) Vessel image of contrast enhanced US. The splenic vein (SV) was enhanced at the dorsal side of the pancreatic body. Vessels in the mass were observed as those in the pancreatic body. (D) Perfusion image of contrast enhanced US. As the mass in the pancreatic head was enhanced homogeneously as in the other part of pancreas, there was no visible tumour-pancreas contrast.

(67\%) of all pancreatic ductal carcinomas (29 type II; one type III; three type IV), and most were relatively hypovascular compared with the surrounding pancreatic tissue (type II).
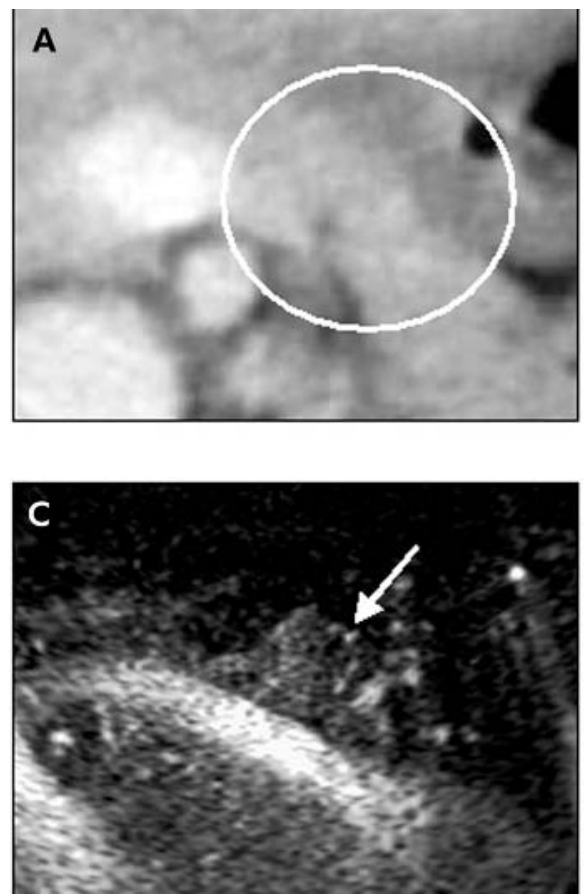

Table 1 shows the sensitivities of contrast enhanced CT, fundamental B-mode US, and contrast enhanced US in depicting pancreatic tumours. Fundamental B-mode US failed to depict six tumours (five ductal carcinomas and
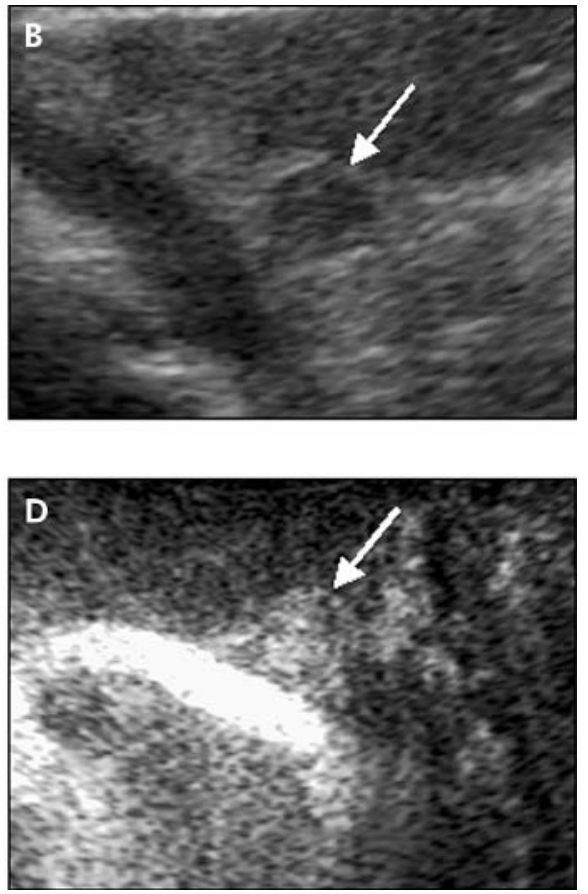

Figure 6 Typical case of a type IV tumour. A 38 year old woman with a pancreatic islet tumour. (A) Contrast enhanced computed tomography does not depict any tumours in the pancreas. (B) Fundamental B-mode ultrasonography (US) shows a low echoic tumour (arrow) of $0.7 \mathrm{~cm}$ in diameter at the pancreatic tail. (C) Vessel image of contrast enhanced US. There were abundant vessels in a spherical tumour (arrow) at the pancreatic tail.

(D) Perfusion image of contrast enhanced US. The tumour (arrow) was relatively hypervascular compared with the other part of the pancreas. 
Table 1 Sensitivity in depicting pancreatic tumours

\begin{tabular}{|c|c|c|c|}
\hline & \multicolumn{3}{|l|}{ Tumour size } \\
\hline & $2 \mathrm{~cm}$ or less & $\begin{array}{l}\text { More than } \\
2 \mathrm{~cm}\end{array}$ & Total \\
\hline $\begin{array}{l}\text { Contrast enhanced CT } \\
\text { Endosonography } \\
\text { Fundamental B-mode US } \\
\text { Contrast enhanced US }\end{array}$ & $\begin{array}{l}13 / 19(68 \%) \\
18 / 19(95 \%)^{*} \\
16 / 19(84 \%) \\
18 / 19(95 \%)^{*}\end{array}$ & $\begin{array}{l}45 / 46(98 \%) \\
45 / 46(98 \%) \\
43 / 46(93 \%) \\
44 / 46(96 \%)\end{array}$ & $\begin{array}{l}58 / 65(89 \%) \\
63 / 65(97 \%) \\
59 / 65(91 \%) \\
62 / 65(95 \%)\end{array}$ \\
\hline $\begin{array}{l}\text { US, ultrasonography; } C T \text {, } \\
{ }^{*} p<0.05 \text { compared with }\end{array}$ & $\begin{array}{l}\text { computed tomo } \\
\text { contrast enhanc }\end{array}$ & $\begin{array}{l}\text { ography. } \\
\text { ced CT. }\end{array}$ & \\
\hline
\end{tabular}

one endocrine tumour) while contrast enhanced CT failed to detect seven tumours. Three of the six tumours that fundamental B-mode US failed to depict were clearly detected by subsequent contrast enhanced US. Although a subabnormality such as stenosis of the main pancreatic duct and swelling of the pancreas was demonstrated on fundamental B-mode US, the tumour was unclear in those three patients. Subsequent contrast enhanced US clearly demonstrated the outline of the tumour (fig 4). Five tumours were seen on contrast enhanced US but not on contrast enhanced CT. They had been suspected on fundamental B-mode US before contrast enhanced US. Nineteen tumours were $2 \mathrm{~cm}$ or less in size. Contrast enhanced CT failed to depict six of these 19 tumours while contrast enhanced US failed to depict only one tumour (table 1). Sensitivity for depicting these 19 tumours on contrast enhanced US was similar to that of EUS (table 1).

Table 2 shows the correlation of the vascular pattern of pancreatic tumours between contrast enhanced CT and contrast enhanced US. When type I and II tumours were regarded as hypovascular, type III as isovascular, and type IV as hypervascular, the vascularity of the tumours depicted by contrast enhanced US was closely correlated with that depicted by contrast enhanced CT. On both contrast enhanced CT and contrast enhanced US, 43 of the 49 ductal carcinomas manifested hypovascular tumours and 14 of the other 16 tumours were isovascular or hypervascular. Values for specificity in diagnosing pancreatic ductal carcinomas with both modalities were $94 \%$. In terms of sensitivities in diagnosing pancreatic ductal carcinomas, there were no significant differences between the two modalities (contrast enhanced US 90\%; contrast enhanced CT 82\%).

\section{DISCUSSION}

Contrast enhanced sonography was first reported with the use of carbon dioxide microbubbles. ${ }^{16}{ }^{17}$ The technique is a sensitive and accurate tool for differentiating pancreatic ductal carcinomas from chronic pancreatitis and pancreatic endocrine tumours. However, it requires an angiographic technique that is relatively invasive because carbon dioxide microbubbles are selectively infused into the coeliac artery or superior mesenteric artery. Levovist, a sonography contrast agent that is infused intravenously, was developed concurrently and is known to be well tolerated with a fairly good safety profile. ${ }^{15}$ However, several limitations with microbubble agents in Doppler US studies, such as blooming artefacts, poor spatial resolution, and low sensitivity to slow flow, were inevitable. ${ }^{1-9}$ The recently introduced phase inversion (pulse inversion) harmonic US technique is a microbubble specific approach that depicts signals from microbubbles sensitively with good spatial resolution devoid of Doppler related artefacts. ${ }^{18}$ However, the technique of phase inversion harmonics does not have the advantage of observation in real time. With concurrent use of the newly developed encoding technology in addition to phase inversion harmonics, signals from microbubbles are much more evident. ${ }^{10-15}$ Furthermore, the encoding technology has made it possible to observe the flow of the bubbles in a real time fashion (vessel image). We observed tumour vessels on real time continuous imaging and tumour parenchymal flow on interval delay scanning by the use of coded phase inversion harmonic imaging. In the present study, fine vessels surrounding the tumour and flowing from the periphery to the centre of the tumour in a real time fashion were visualised in $67 \%$ of all pancreatic ductal carcinomas. This pathophysiological phenomenon of pancreatic tumours was first visualised by means of this novel technology.

It is not difficult to manipulate contrast enhanced coded phase inversion harmonic US. Reliable imaging can be obtained after conducting this examination in 10-20 cases. The sonographer (KM) in the present study had conducted contrast enhanced US in more than 1000 patients before beginning the present study. The reviewers (MK and TK) had experience of more than 300 cases of reading images of contrast enhanced US. Therefore, reliable imaging and reading were performed throughout our study. On the basis of the patterns of vessel and perfusion images of contrast enhanced US, we classified tumour vascularity into four patterns. We calculated contrast indices to confirm the reliability of the classification by the reviewers, although they were obtained only from the perfusion images. The fact that the contrast indices sequentially increased from type I to type IV supports the reliability of the classification by the reviewers. Ductal carcinoma is known to be a hypovascular tumour. The typical vascular patterns of the tumour manifest low attenuation relative to the surrounding pancreatic tissue on contrast enhanced CT which is known to be useful in diagnosing pancreatic diseases. ${ }^{19}{ }^{20}$ When type I and II tumours together are categorised as a hypovascular pattern, most ductal carcinomas in the present study were hypovascular on contrast enhanced US while the other tumours were isovascular or hypervascular. The fact that the vascular patterns of the tumours on contrast enhanced US correlated well with those on contrast enhanced CT suggests that contrast enhanced US is as reliable as contrast enhanced CT for diagnosing pancreatic tumours.

Contrast enhanced US enabled depiction of the margin of the three tumours that fundamental B-mode US failed to detect, suggesting that visualisation of vascularity may assist

Table 2 Comparison of vascularity of pancreatic tumours between contrast enhanced computed tomography (CT) and contrast enhanced ultrasonography (US)

\begin{tabular}{lllllll}
\hline & & \multicolumn{3}{l}{ Image patterns of contrast enhanced US } \\
\cline { 3 - 7 } & I & II & III & IV & ND \\
\hline Contrast enhanced CT & Hypovascular & 14 & 25 & & & 1 \\
& $\begin{array}{l}\text { Isovascular } \\
\text { Hypervascular }\end{array}$ & & & 8 & 9 & 1 \\
& ND & 1 & 4 & & 1 & 1 \\
\hline
\end{tabular}

ND, Not detected 
in discriminating tumours from the surrounding tissue in US examinations. In addition, contrast enhanced US depicted six tumours that contrast enhanced CT failed to depict. In particular, the sensitivity of contrast enhanced US (95\%) in depicting small tumours of $2 \mathrm{~cm}$ or less in size was remarkably different from that of contrast enhanced CT $(68 \%)$. These results indicate that contrast enhanced US may be superior to contrast enhanced CT in depicting small tumours due to higher spatial resolution. Alternatively, contrast enhanced US may more clearly discriminate blood flow in the pancreatic tumour from that in the surrounding tissue. With regard to depicting ability, contrast enhanced US was similar to EUS, which is known to be superior to any other modalities with respect to spatial resolution. ${ }^{21}$

In conclusion, this new technique made it possible to depict fine vessels surrounding the tumour and flowing from the periphery to the centre of the tumour in a real time fashion, facilitating differential diagnosis of pancreatic tumours. To the best of our knowledge, there have been no reports that have shown the architecture of fine vessels in pancreatic cancers other than by sonography. In addition, another advantage of contrast enhanced US is that it improved the depiction of small lesions compared with fundamental B-mode US and contrast enhanced CT. Further prospective controlled studies are required where magnetic resonance imaging, EUS, and contrast enhanced harmonic US should be compared with the gold standard operation and histology.

\section{ACKNOWLEDGEMENTS}

The present study was supported by grants from the Japan Society for the Promotion of Science.

\section{Authors' affiliations \\ M Kitano, M Kudo, Y Suetomi, H Sakamoto, N Fukuta, R Nakaoka,}

T Kawasaki, Division of Gastroenterology and Hepatology, Department of Internal Medicine, Kinki University School of Medicine, Ohno-Higashi, Osaka-Sayama, Japan

K Maekawa, Section of Abdominal Ultrasound, Kinki University School of Medicine, Ohno-Higashi, Osaka-Sayama, Japan

\section{REFERENCES}

1 Ueno N, Tomiyama T, Tano S, et al. Contrast enhanced color Doppler ultrasonography in diagnosis of pancreatic tumor: two case reports. $J$ Ultrasound Med 1996;15:527-30.
2 Bhutani MS, Hoffman BJ, Velse A, et al. Contrast-enhanced endoscopic ultrasonography with galactose microparticles:SHU508A (Levovist). Endoscopy 1997;29:635-9.

3 Ricke J, Hänninen LE, Amthauer $\mathrm{H}$, et al. Assessment of the vascularisation of endocrine tumors by stimulated acoustic emission of SHU 508A ultrasound contrast agent and color or power Doppler sonography. Invest Radiol 2000;4:253-9.

4 Becker D, Strobel D, Bernatik T, et al. Echo-enhanced color- and powerDoppler EUS for the discrimination between focal pancreatitis and pancreatic carcinoma. Gastrointest Endosc, 2001;53:784-9.

5 Ding H, Kudo M, Onda H, et al. Hepatocellular carcinoma: depiction of tumor parenchymal flow with intermittent harmonic power Doppler US during the early arterial phase in dual-display mode. Radiology 2001;220:349-56.

6 Ding H, Kudo M, Maekawa K, et al. Detection of tumor parenchymal blood flow in hepatic tumors: value of second harmonic imaging with a galactosebased contrast agent. Hepatol Res 2001;21:242-51.

7 Ding H, Kudo M, Onda H, et al. Sonographic diagnosis of pancreatic islet cell tumor: value of intermittent harmonic imaging. J Clin Ultrasound 2001;29:411-16.

8 Ding $\mathrm{H}$, Kudo $M$, Onda $\mathrm{H}$, et al. Contrast-enhanced subtraction harmonic sonography for evaluating treatment response in patients with hepatocellular carcinoma. AJR 2001;176:661-6.

9 Rickes S, Unkrodt K, Neye H, et al. Differentiation of pancreatic tumours by conventional ultrasound, unenhanced and echo-enhanced power Doppler sonography. Scand J Gastroenterol 2002;37:1313-20.

10 Ding H, Kudo $M$, Onda $H$, et al. Evaluation of post-treatment response of hepatocellular carcinoma with contrast-enhanced coded phase-inversion harmonic US: comparison with dynamic CT. Radiology 2001;221:721-30.

11 Minami Y, Kudo M, Kawasaki T, et al. Evaluation of the effectiveness of transcatheter arterial chemoembolization for hepatocellular carcinoma: value of coded phase-inversion harmonics. AJR 2003;180:703-8.

12 Wen YL, Kudo M, Minami Y, et al. Radiofrequency ablation of hepatocellular carcinoma: therapeutic response using contrast-enhanced coded phaseinversion harmonic sonography. AJR 2003;181:57-63.

13 Wen YL, Kudo M, Zheng RQ, et al. Characterization of hepatic tumors: value of contrast-enhanced coded phase inverstion harmonic US. AJR 2004 (in press).

14 Minami Y, Kudo M, Kawasaki T, et al. Radiofrequency ablation for sonography undetectable for hepatocellular carcinoma in the hepatic dome: value of contrast-enhanced harmonic sonography associated with artificial pleural effusion. AJR 2004 (in press).

15 Kudo M. Contrast harmonic imaging in the diagnosis and treatment of hepatic tumors. Tokyo: Springer, 2003.

16 Kato T, Tsukamoto Y, Naitoh Y, et al. Ultrasonographic and endoscopic ultrasonographic angiography in pancreatic mass lesions. Acta Radiologica 1995;36:381-7.

17 Koito K, Namieno T, Nagakawa T, et al. Inflammatory pancreatic masses: Differentiation from ductal carcinomas with contrast-enhanced sonography using carbon dioxide microbubbles. AJR 1997;169:1263-7.

18 Oshikawa O, Tanaka S, loka $\mathrm{T}$, et al. Dynamic sonography of pancreatic tumors: comparison with dynamic CT. AJR 2002;178:1133-7.

19 Diehl SJ, Lehman KJ, Sandick M, et al. Pancreatic cancer: value of dual-phase helical CT in assessing resectability. Radiology 1998;206:373-8.

20 Boland GW, O'Malley ME, Saez M, et al. Pancreatic-phase versus portal vein-phase helical $C T$ of the pancreas: optimal temporal window for evaluation of pancreatic adenocarcinoma. AJR 1999;172:605-8.

21 Zuccaro G, Sterling MJ. Endosonography in pancreatic disease: Differential diagnosis. In: van Dam J, Sivak MV, eds. Gastrointestinal Endoscopy. Philadelphia: Saunders, 1999:235-43. 